\title{
Les Malacocénoses de quatre lacs haut-alpins
}

\author{
J. Mouthon 1
}

Au cours d'une prospection malacologique de quatre lacs haut-alpins (altitude $2000 \mathrm{~m}$ ) et d'un ruisseau affluent, 7 espèces de mollusques, 5 bivalves et 2 gastèropodes ont été inventoriées.

Une analyse de la répartition des peuplements dans les zones littorales, sublitiorales et profondes est effectuée. Les malacocénoses répertoriées correspondent aux groupements caractéristiques du cours supérieur des écosystèmes d'eau courante et apparaissent comme très sensibles à une augmentation même locale de la minéralisation des eaux.

The molluscs of four high-alpine lakes.

In a survey of the molluscs of four high-alpine lakes (altitude $2000 \mathrm{~m}$ ) and a tributary stream, seven species were recorded; five bivalves and two gastropods.

An analysis was made of the distribution of the populations in the littoral, sublittoral and profundal zones. The mollusc cenoses are similar to groups characteristic of the upper reaches of rivers and appear to be very sensitive to local increases in the mineralisation of the water.

Les études écologiques d'écosystèmes lacustres d'altitude $(2000 \mathrm{~m})$ sont peu nombreuses. En France seuls quelques lacs des massifs d'Estibère et de Néouvielle comme le lac de Port Bielh dans les Pyrénées ont fait l'objet d'un inventaire faunistique plus ou moins détaillé (Angelier 1961, Laville 1972, Giani et Laville 1973, Juget et Giani 1974). Toutefois certains groupes de macro-invertébrés comme les mollusques n'ont pas été étudiés.

En ce qui concerne la faune malacologique, les données que l'on possède se rapportent uniquement aux populations des zones littorales de plusieurs lacs et étangs du massif du Carlit dans les Pyrénées. (Kuiper 1964, Combes et al 1971) et des Alpes (Blanchard et Richard 1897, Coutagne 1929, Jayet 1973. Kuiper 1974).

A la demande du Ministère de l'Environnement et dans le but de dégager des propositions de gestion des milieux lacustres d'altitude, une diagnose écologique de quatre plans d'eau alpins (Rapport CEMAGREF 1983) a été entreprise. Nous présentons ici les résultats d'une prospection malacologique de ces lacs.

1. Laboratoise d'hydrobiologie du CEMAGREF, 3. quai Chaureau, 69009 Lyon.

\section{1. - Cadre topographique}

Les quatre lacs étudiés se trouvent dans le département des Hautes-Alpes, au Nord de Briançon et à l'Est des cols du Galibier et du Lautaret.

Le lac du Grand Ban, les lacs Rond et de la Clarée, petite pièce d'eau dans laquelle aucun mollusque ne fut découvert, se succèdent au pied du col des Rochilles. Ces plans d'eau étant séparés par des barrages d'éboulis plus ou moins perméables, le Grand Ban se vide dans le lac Rond qui à son tour alimente partiellement la source de la Clarée.

Surplombant la vallée de Névache on trouve le lac du Serpent dont une partie des eaux alimente le lac Laramon situé en contrebas, l'exutoire de ce dernier allant grossir la Clarée, impétueux torrent qui draine les eaux de cette vallée. A l'inverse des écosystèmes précédents leur niveau demeure stable.

Ces lacs, dont les principales caractéristiques morphologiques figurent dans le tableau 1 , sont totalement dépourvus de ceinture végétale.

\begin{tabular}{lcrcc}
\hline & $\begin{array}{c}\text { Lac } \\
\text { Grand Ban }\end{array}$ & $\begin{array}{c}\text { Lac } \\
\text { Rond }\end{array}$ & $\begin{array}{c}\text { Lac du } \\
\text { Serpent }\end{array}$ & $\begin{array}{c}\text { Lac } \\
\text { Laramon }\end{array}$ \\
\hline Altitude (m) & 2450 & 2380 & 2450 & 2360 \\
Long. max. (m) & 300 & 435 & 250 & 450 \\
Larg. max. (m) & 160 & 210 & 185 & 175 \\
Prof. max. (m) & 10 & 9 & 10 & 12 \\
\hline
\end{tabular}

Tableau I. Principales caractéristiques morphologiques. 


\section{2. - Données géologiques et physico- chimiques}

Pendant la période de dégel, on constate l'existence d'une stratification thermique inverse. Au cours de l'été les températures maximales ${ }^{1}$ de ces quatre lacs demeurent basses; Grand Ban $14^{\circ} 3$, Lac Rond $12^{\circ} 2$, Serpent $12^{\circ} 5$, Laramon $12^{\circ} 2$ et sans doute en partie à cause de leur faible profondeur le brassage des eaux semble permanent (cf. rapport CEMAGREF 1983).

L'évolution, d'avril à septembre, de l'oxygène dissous révèle un déficit important des concentrations de ce paramètre en fin de période de dégel, dans la zone profonde des lacs du Serpent et Laramon (fig. 1). Il en est probablement de même pour le Grand Ban et le Lac Rond pour lesquels les mesures du printemps n'ont pu être réalisées, la masse d'eau étant semble-t-il totalement prise par les glaces.

Les quatre lacs étudiés sont disséminés sur les enveloppes sédimentaires des zones briançonnaise et subbriançonnaise. Le bassin versant surplombant la vallée glaçiaire où s'étalent les lacs du Grand Ban

1. Températures relevées en surface le 03/09/80.
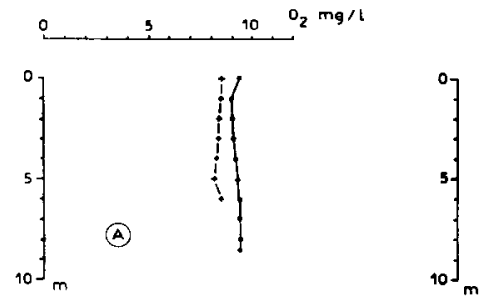

(B)

et Rond est surtout constitué par des terrains d'âge triasique calcaréo-dolomitiques. De ce fait leurs eaux sont plus minéralisées et riches en alcalinoterreux, mais cependant plus pauvres en sulfates que celles des lacs Laramon et du Serpent environnés par les couches du Houiller (grès, schistes pélitique, conglomèrats à galets de quartz et micaschistes). Toutefois les concentrations en sels dissous de ces eaux demeurent plutôt réduites (Tableau II), par conséquent ces milieux lacustres peuvent être clas. sés dans les écosystèmes qualifiés d'oligotrophes (Vollenweider 1975).

\section{3. - Techniques d'échantillonnage}

Les mollusques de la zone littorale et de l'afférence ont été récoltés à l'aide d'un troubleau à base rectangulaire $($ Long $=25 \mathrm{~cm}$, Haut $=18 \mathrm{~cm})$. L'échantillonnage réalisé sur toute la largeur de la zone littorale représente une surface totale de 1, 2, parfois $3 \mathrm{~m}^{2}$ par station. Les zones profondes ont été prospectées à l'aide d'une Benne Petersen (surface $=350 \mathrm{~cm}^{2}$ ), trois à six dragages étant effectués à chaque point d'échantillonnage.
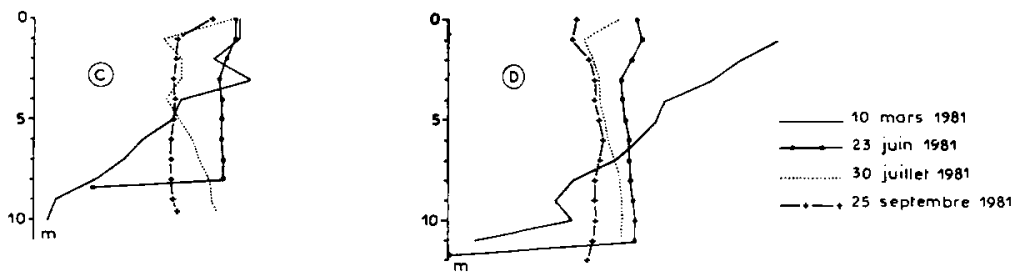

Fig. 1. Evolution des concentrations de l'oxygène dissous. A. Grand Ban - B. Rond - C, Serpent - D, Laramon. 
Tableau II. Données physico-chimiques, analyses du 3/9/1980.

\begin{tabular}{|c|c|c|c|c|c|c|c|c|c|c|c|c|c|c|c|}
\hline & Lac & Grano & Ban & & Ic Rond & & & $\operatorname{Lac} d u$ & Serper & & & ac lar & amon & & \\
\hline & Om & -3 & $\begin{array}{c}\text { Fond } \\
-6\end{array}$ & 0 & -3 & $\begin{array}{c}\text { Fond } \\
-6\end{array}$ & 0 & -3 & -6 & $\begin{array}{l}\text { Fond } \\
-9\end{array}$ & 0 & -3 & -7 & $\begin{array}{l}\text { Fond } \\
=-12\end{array}$ & $\begin{array}{l}\text { Affe- } \\
\text { rence }\end{array}$ \\
\hline$\theta^{\circ} \mathrm{c}$ & 9,8 & 9,4 & 9 & 8,3 & 7,7 & 5,5 & 8,6 & 8,2 & 7,9 & 7,7 & 8,9 & 8,9 & 8,8 & 8,7 & 10,3 \\
\hline$P h$ & 8,4 & 8,3 & 8,4 & 8,1 & 8,1 & 8,1 & 8,1 & 7,5 & 7,4 & 7,8 & 7,2 & 7,3 & 8,1 & 7,3 & 8,4 \\
\hline Cus & 118 & 118 & 119 & 135 & 136 & 145 & 72 & 72 & 71 & 72 & 66 & 65 & 65 & 65 & 84 \\
\hline $\begin{array}{l}\text { Oxyd. } \\
\mathrm{mg} / 1\end{array}$ & 0,31 & 0,26 & $<0,1$ & 0,33 & 0,30 & 0,23 & 0,2 & 0,02 & 0,44 & 0,05 & 0,24 & 0,76 & 0,94 & 3,2 & 1,51 \\
\hline $\mathrm{NH}_{4}{ }^{+}$ & 0,15 & 0,10 & 0,26 & 0,08 & 0,08 & 0,06 & 0,06 & 0 & 0,026 & 0,23 & 0,08 & 0,01 & 0,05 & 0,01 & 0,026 \\
\hline $\mathrm{NO}_{2}-$ & 0,03 & 0 & 0 & 0 & 0 & 0 & 0 & 0.07 & 0,03 & 0,03 & 0 & 0 & 0 & 0 & 0 \\
\hline $\mathrm{NO}_{3}-$ & 0 & 0,09 & 0 & 0 & 0,09 & 0,18 & 0,22 & 0 & 0,18 & 0.75 & 0,09 & 0,04 & 0,27 & 0 & 0,27 \\
\hline $\mathrm{PO}_{4} *-$ & 0 & 0 & 0 & 0 & 0 & 0 & 0 & 0 & 0 & 0 & 0 & 0 & 0,13 & 0,13 & 0 \\
\hline $\mathrm{S}_{102}$ & 0,2 & 0,2 & 0,5 & 0,3 & 0,4 & 0,5 & 2 & 1,8 & 2 & 2 & 1,6 & 1,5 & 1,5 & $1 ; 5$ & 3 \\
\hline $\mathrm{HCO}_{3}^{-}$ & 64 & 67 & 61 & 73 & 73 & 73 & 30 & 31 & 31 & 31 & 28 & 27 & 31 & 27 & 31 \\
\hline $\mathrm{CL}=$ & $<0,5$ & $<0,5$ & $<0,5$ & 4,5 & $<0.5$ & $<0,5$ & $<0,5$ & $<0,5$ & $<0,5$ & $<0,5$ & $<0,5$ & $<0,5$ & $<0,5$ & $<0.5$ & $<0.5$ \\
\hline $504^{--}$ & 0 & 0 & 0 & i & 0 & 0 & 6 & 5 & 5 & 6 & 5 & 4 & 5 & 5 & 3 \\
\hline $\mathrm{Ca}^{++}$ & 18 & 18 & 18,4 & 19 & 19,6 & 19,6 & 11 & 11 & 9 & 9 & 9 & 8.6 & 8,6 & 8,6 & 11.2 \\
\hline $\mathrm{Mg}^{++}$ & 3,3 & 3,4 & 3,4 & 3,5 & 3,4 & 4 & 2,4 & 2,4 & 2,4 & 2,4 & 2,4 & 2,1 & 2,1 & 2 & 2 \\
\hline$k^{+}$ & $<0,05$ & 0,08 & $<0,05$ & 0,16 & 0,12 & 0,2 & 0,32 & 0,28 & 0,32 & 0,32 & 0,32 & 0,28 & 0,32 & 0.6 & 0,36 \\
\hline
\end{tabular}

L'abondance des espèces prêlevées est ensuite rap. portée au $\mathbf{m}^{2}$.

Les échantillons furent tamisés à $450 \mu$ et triés sous la loupe binoculaire.

Les prélèvements réalisés au cours du mois de juillet 1981 concernent 25 points répartis dans les zones littorales et profondes de ces quatre lacs.

\section{4. - Les Malacocénoses}

\section{1. - Répartition des espèces dans la zone litto- rale $(0 \quad 1,5 \mathrm{~m})$}

a) Les lacs Rond et du Grand Ban

Compte tenu des variations importantes du niveau des eaux que subissent ces deux écosystemes, leur remplissage étant maximal pendant la période du prélèvement, aucun mollusque n'a pu être récolté dans leur zone littorale.

b) Le lac du Serpent

L'essentiel de la faune malacologique de ce lac est constitué par deux espèces de Sphaeriidae: Pisidium casertanum et Pisidum hibernicum, inégalement réparties dans la zone rivulaire (tableau 3 et fig. 2). Seuls deux spécimens de Lymnaea truncatula ont pu être récoltés au voisinage de l'arrivée de petits ruisselets de drainage à fond riche en matières organiques (Point 1) dans lesquels cet te espèce se développe préférentiellement.

L'abondance brute des espèces de pisidies varie remarquablement d'un point d'échantillonnage à l'autre. Elle est minimale au point 4 , au niveau duquel se trouve une beine réduite, pentue, constituée par une granulométrie grossière où débouche un ruisseau à écoulement torrentiel, aux eaux fraîches et peu minéralisées. Lorsque la zone littorale s'élargit les sédiments fins prédominent et l'abondance augmente (points 2 et 3). Cependant c'est au voisinage de l'arrivée dans le lac de plusieurs ruisselets à écoulement lent, drainant sa bordure marécageuse et provoquant sans doute un enrichissement local du milieu en substances dissoutes, que la densité des deux espèces de pisidies répertoriées (cf. Lac Laramon) est maximale (Point 1).

\section{c) Lac Laramon}

Quatre espèces de mollusques ont été recensées dans la zone littorale de ce plan d'eau; une espèce de Gastéropode, Lymnaea peregra, et trois espèces de Sphaeriidae : Pisidium nitidum venant s'ajouter aux deux pisidies peuplant le lac précédent (Tableau III). 

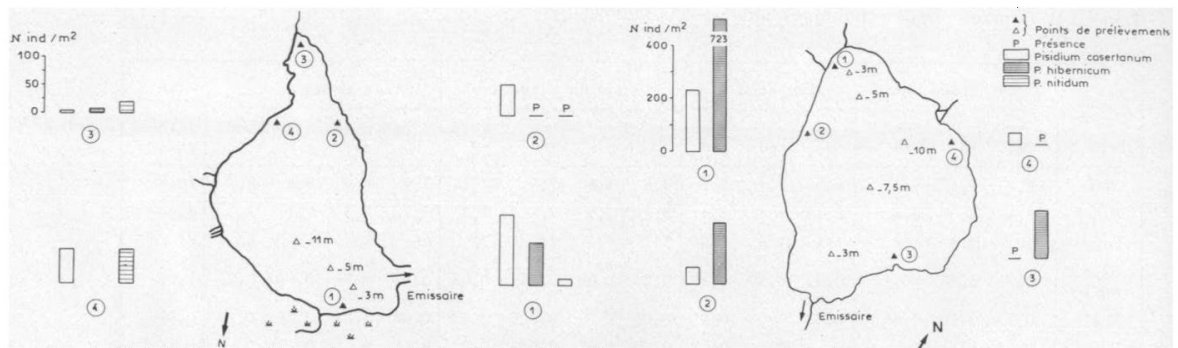

Fig. 3. Distribution de l'abondance $\left(\mathrm{m}^{2}\right)$ des Sphaeriidae dans la zone littorale du lac Laramon et emplacement aes points d'échantillonnage dans les zones sublit torales et profondes.

Tableau III. Abondance au $\mathrm{m}^{2}$ des espèces de mollusques aux différentes profondeurs prospectées.

\begin{tabular}{|c|c|c|c|c|c|c|}
\hline Profondeur (a) & & $\begin{array}{l}\text { Lae Grand Ban } \\
0-5 ;-8:-10\end{array}$ & $\begin{array}{l}\text { Lee Rond } \\
0-1,5 ;-2 ;-3 ;-4 ;-9\end{array}$ & $\begin{array}{l}\text { Lac du Serpent } \\
0-1:-3:-5:-7.5:-10\end{array}$ & $\begin{array}{l}\text { Lec Laramon } \\
0-1:-3:-5:-11\end{array}$ & $\begin{array}{l}\text { Af } \mathrm{ft}- \\
\text { rence }\end{array}$ \\
\hline Lumnaed peregma & (Mu1ler) & & & & 15 & \\
\hline Lymmaea trieicatuia & Mullet & & & 2 & & \\
\hline Pigidium arsertanum & (Poli) & $996: 776$ & 760 & $112: 24: 16$ & 78 & 1070 \\
\hline Piaidiun hibermitewn & Weoterlund & 8 & 24 & $359: 40$ & $72: 24,64$ & \\
\hline Piaidium nititum & Jenyns & & & $i$ & 23 & \\
\hline Pigidium personatum & $\operatorname{Ma} 1 \mathrm{~m}$ & & & $i$ & $i$ & 32 \\
\hline Piaidiven aubtrunoditum & Mala & & 116 & $i$ & 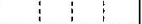 & \\
\hline $\mathrm{Nb}=\mathrm{p}$ & & i & 2 & 1 & 4 & 2 \\
\hline Nb ind $/ \mathrm{m}^{2}$ & & 996 & $-460900,48$ & \begin{tabular}{l|l|l|l}
473 & 64 & 24 & 8
\end{tabular} & $188: 24: 80: 4$ & 2102 \\
\hline
\end{tabular}

L'abondance brute des bivalves est maximale au point 1 où conflue un ruisseau drainant une zone marécageuse à fond riche en limon et en matières organiques, aux eaux plus minéralisées que celles du lac lui-même (Tableau II), et dans lequel prolifèrent Pisidium casertanum et Pisidium personatum. En outre, on constate que c'est également à cette extrémité du plan d'eau que Lymnaea peregra atteint sa densité maximale : $15 \mathrm{Ind} / \mathrm{m}^{2}$ (fig. 3 ).

Pisidium nitidum, peu représentée dans les sédiments tourbeux (point 2), atteint une abondance notable au point 4 où la granulométrie (sableuse) est plus grossière qu'au point 1 (sablo-limoneuse).

\section{2. - Distribution bathymétrique}

En raison du marnage important qui affecte le niveau de leurs eaux, c'est respectivement à partir de $5 \mathrm{~m}$ dans le Grand Ban et de $\mathbf{2} \mathrm{m}$ dans le lac Rond (lorsque leur remplissage est optimum) que l'on ren- contre quelques spécimens de Pisidium casertanum (fig. 4, 5 et 6). C'est cette espèce, sans doute la plus rustique, la plus résistante au gel et à la dessication parmi les pisidies (Favre 1927 ; Kuiper 1966 ; Danneel et Hinz 1974) qui colonise les fonds particulièrement exposés des zones rivulaires de ces deux lacs. En revanche Pisidium hibernicum et Pisidium subtruncatum vivent plus en profondeur puisqu'elles n'apparaissent qu'aux environs de $8 \mathrm{~m}$ dans le Grand Ban et de $3 \mathrm{~m}$ dans le lac Rond (fig. 6).

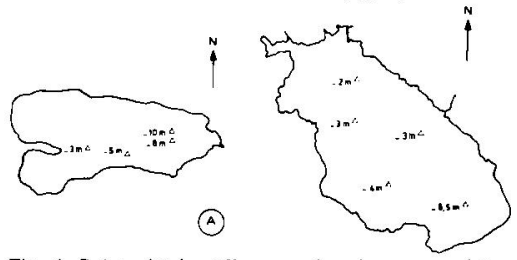

Fig. 4. Points d'échantillonnage dans les zones sublitto. rales et profondes des lacs Grand Ban (A) et Rond (B). 


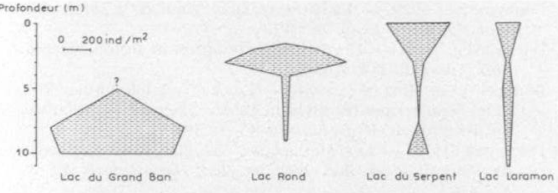

Fig. 5. Distribution bathymétrique des mollusques.

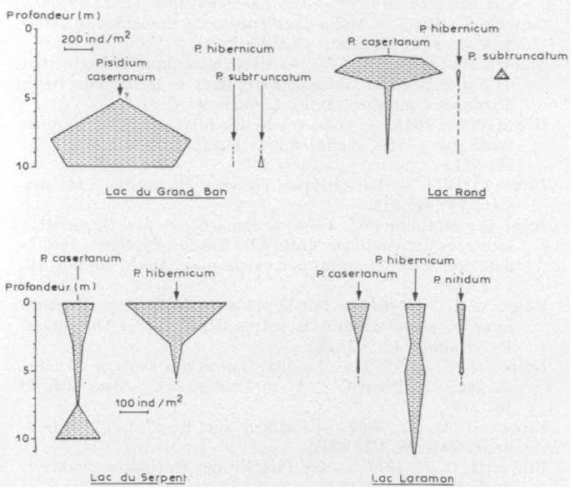

Fig. 6. Distribution bathymétrique des différentes espèces de mollusques.

A l'exception du Grand Ban où règnent des conditions très particulières (marnage important) on enregistre, pour les trois autres lacs une diminution importante de l'abondance des espèces en fonction de la profondeur probablement due aux trop faibles concentrations en oxygène dissous régnant au voisinage des fonds, notamment en fin de période hivernale (fig. 1, 5 et 6).

L'abondance maximale atteinte par Pisidium casertanum dans les fonds du lac du Serpent pourrait être liée à des arrivées d'eau sous-lacustres; c'est peut-être également le cas dans le Grand Ban. En revanche sa disparition à partir de $5 \mathrm{~m}$ dans le lac Laramon est surprenante.

\section{5. - Remarques et discussion}

Les spécimens de Lymnaea peregra du Lac Laramon présentent une coquille aux premiers tours de spire généralement très érodés, moins globuleuse que les exemplaires de plaine mais de longueur maximale voisine (environ $19 \mathrm{~mm}$ ); ils appartiennent à la variété nivalis (in Germain 1931).

Les espèces de Sphaeriidae récoltées possèdent une coquille mince, fragile, une chamière étroite, et, particulierement en ce qui concerne Pisidium casertanum et $P$. hibemicum dont la forme particulière a été qualifiée de giganteum (Favre 1927), atteignent une taille bien supérieure à celle de leurs voisines de plaine.

Ces bivalves présentent donc un ensemble de caractéristiques particulières, déjà décrites par d'autres auteurs (Jayet 1973, Kuiper 1974), propres à la fois aux milieux de hautes altitudes et à la zone circumbboréale (Kuiper 1964).

Les valves de pisidies montrent des arrêts de croissance nets, fortement marqués, mais ces « annuli " ne permettent pas de tirer des conclusions quant à la Jongévité de ces bivalves (Meier-Brook 1970, Heard 1977, Kuiper 1983).

Toutefois, comme l'a constaté Jayet (1973), les irrégularités de croissance des pisidies des milieux hautalpins ne déterminent pas de profil irrégulier en zig zag des valves, comme cela a pu être observé sur des exemplaires actuels de Pisidium obtusale Clessin notamment du nord de la Suede (Odhner 1951; Dance 1962), de Sibérie (Kuiper 1969), ou fossiles du pleistocène récent (Favre et Jayet 1938).

Il est par ailleurs surprenant de constater que Pisidium conventus Clessin, espèce sténotherme d'eau froide à distribution " nordique pré-alpine " abondante dans la zone littorale des milieux lacustres nordiques (Stelfox et al. 1972, Kuiper 1974) etPisidium lilljeborgii (Clessin) à distribution alpine et holarctique (Kuiper 1966), abondante dans plusieurs lacs du Jura (Mouthon 1981b) sont absentes des zones littorales et profondes de ces écosystèmes dans lesquels on pourrait s'attendre à les rencontrer.

Comme on l'a observé dans les lacs du Serpent et de Laramon, gastéropodes et sphaeriidae sont très sensibles à une augmentation même locale de la minéralisation des eaux se traduisant par un accroissement remarquable de l'abondance de ces espèces. Ce phénomène déjà observé lors de diagnoses écologiques de plusieurs lacs jurassiens (Mouthon op. cit.) montre l'intérêt d'une analyse quantitative de la distribution des mollusques dans l'ap- 
préciation de l'impact des afférences et des rejets en milieu lacustre.

Les malacocènoses des quatre lacs haut-alpins composées de : Pisidium subtruncatum, Pisidium nitidum et Lymnaea peregra correspondent aux groupements caractéristiques du cours supérieur (Rhithron supérieur et moyen) des écosystèmes d'eaux courantes (Mouthon 1981a). Seule Pisidium hibernicum, espèce plutôt eurytope et généralement peu abondante dans les eaux courantes (Kuiper 1966), mais que j'ai récoltée en abondance dans deux petites rivières aux eaux peu minéralisées, La Moine (département Loire-Atlantique) et Le Rahin (dépar. tement Haute-Saône), aux niveaux de seuils réduisant notablement la vitesse du courant, semble faire exception.

Des recherches sont en cours, visant à vérifier si il existe réellement une identité entre la distribution longitudinale théorique des mollusques dans les écosystèmes d'eaux courantes (Mouthon op. cit.) et les malacocénoses de milieux lacustres parvenus à différents degrés de trophie.

\section{Remerciements}

Je remercie Monsieur J. G. J. Kuiper (Institut Neerlandais, Paris). le Personnel du Laboratoire d'Hydrobiologie d'Aix-en-Provence et particulierement Messieurs Dumont B. et Rivier B. pour l'aide qu'ils mont apportée. Madame Monnier qui s'est chargée de l'éxé. cution des graphiques, et la $31^{\mathrm{e}}$ escadrille d'hélicoptères légers $\left(27^{\mathrm{c}}\right.$ Division alpine) de Talard qui a considérablement facilité nos déplacements.

\section{Travaux cités}

Angelier (E.). 1961. - Les lacs de l'Estibere : remarques sur le peuplement des lass de montagne. Bull. Soc. Hist. Nat. Toulouse, 96 (1/2) : 7-14.

Blanchard (R.) et Richard (J.). 1897. - Sur la faune des lacs élevés des Hautes-Alpes. Mém. Soc. Zool. Fr., 10:43-61.

Combes (CL.). Kuiper (J. G. J.) et De Stefano (Y.). 1971. Ecologie des espèces du genre Pisidium PF. (Mollusques. Sphaeriidae) dans les étangs du Carlit (Pyrénées). La Terre et la Vie, $25: 96-121$.
Coutagne (G.). 1929. - La Faune malacologique de la Tarentaise. Ann. Soc. Linn. Lyon. $74: 7-79$.

Dance (SP.). 1962. - On the genus Pisidium at Upton Warren. Phil. Trans. B. 244 : 418-421.

Danneel (I.) et Hinz (W.). 1974. - Trockenresistenz dreier Pisidium-Populationen (Bivalvia) in Abhängigkeit von der relativen Lufteuchtikeit. Hydrobiologia 45, 1 : 39.43.

Favre (J.). 1927. - Les Mollusques post-glaciaires du Bassin de Genève. Mém. Soc. Phys. et Hist. Nat. Genève. 40, 3: 171434.

Favre (J.) et Jayet (A.). 1938. - Deux gisements post-glaciaires anciens à Pisidium Vincentianum et Pisidium lapponicum aux environs de Genève. Ecl. Geol. Helvetiae 31, 2; 395.402.

Germain (L.). 1931. - Mollusques terrestres et fluviatiles. In Faune de France. 21-22, Lechevalier éd. Paris. $897 \mathrm{p}$.

Giani (N.) et Laville (H.). 1973. - Cycle biologique et production de Sialis lutaria L. (Mégaloptere) dans le lac de Port-Bielh (Pyrénées Centrales). Annls. Limnol, 9: 45-61.

Heard (W.H.). 1965, - Comparative life histories of North American pill Clams (Sphaeriidae: Pisidium). Malacologia 2 : 381411.

Jayet (A.). 1973. - Sur quelques Pisidium Haut-Alpins. Malacologia $14: 415-418$

Juget (J.) et Giani (N.). 1974. - Répartition des Oligochetes lacustres du massif de Néouvielle (Hautes-Pyrénées) avec la description de Peloscolex pyrenaicus n. sp. Annls. Limnol., 10 , $1: 33-53$.

Kuiper (J.G. J.). 1964. - Sur la présence des espèces du genre Pisidium dans les eaux lacustres des Pyrénées Orientales. Vie et Milieu 15:677-685.

Kuiper (J. G. J.). 1966. - La distribution des especes vivantes du genre Pisidium C. P. F. en France. J. Conchyol 105, 4: 181.215 .

Kuiper (J. G. J.). 1959. - Pisidien aus Kasakstan, Sibirien. Arch. Moll. 99, 1/2:49-53.

Kuiper (J. G. J.). 1974. - Die Pisidien der Hochalpengewässer. Arch. Moll. $104: 1.27$.

Kuiper (J. G. J.) 1983. - The Sphaeriidae of Australia. Basteria $47: 3-52$,

Laville (H.). 1972. - Recherches sur les Chironomides (Diptera) lacustres du massif de Néouvielle (Hautes-Pyrénées). 1. Systematique, écologie, phénologie. Annis. Limnol. 7 : 173-332.

Meier-Brook (C.). 1970, - Untersuchungen zur Biologie einiger Pisidium Arten Arch. Hydrobiol, suppl. 38:73-110.

Mouthon (J.). 1981a. - Typologie des Mollusques des eaux courantes. Organisation biotypologique et groupements socioécologiques. Annis. Limnol. $17:$ 143-162.

Mouthon (J.). 1981b. - Les Mollusques des lacs de l'Abbaye et des Rousses (Massif du Jura). Ann. Scient. Univ. Besançon. 4, 2 : (sous presse).

Odhner (N.). 1951. - Swedish high mountain mollusca. In * The mountain fauna of the Virihaure area in Swedish Lapland $\mathrm{s}$. Acta Univ. Lund. (N. S.) $46: 26-50$. Rapport CEMAGREF..

Vollenweider (R. A.). 1975. - Input. Output models, with special reference to the phosphorus loading concept in Limnology. Rev. Suisse Hydrol. 37, 1 : 53-84. 